\title{
Deformed exponentials and logarithms in generalized thermostatistics
}

\author{
Jan Naudts \\ Departement Natuurkunde, Universiteit Antwerpen UIA, \\ Universiteitsplein 1, 2610 Antwerpen, Belgium \\ E-mail: Jan.Naudts@ua.ac.be \\ v3, March 2002
}

\begin{abstract}
Criteria are given that $\kappa$-deformed logarithmic and exponential functions should satisfy. With a pair of such functions one can associate another function, called the deduced logarithmic function. It is shown that generalized thermostatistics can be formulated in terms of $\kappa$-deformed exponential functions together with the associated deduced logarithmic functions.
\end{abstract}

\section{Introduction}

Several proposals exist for generalization of the Boltzmann-Gibbs formalism of equilibrium statistical physics. Examples, considered in the present paper, are Tsallis' thermostatistics [3], and the formalism recently proposed by Kaniadakis [12, 13. In these theories, the logarithm appearing in Shannon's measure of information content is replaced by a deformed logarithmic function [4]. Then the equilibrium distribution is calculated by maximizing information content under the constraint that the average energy is constant. The result is a Boltzmann-Gibbs distribution in which the exponential function is replaced by a deformed exponential function. The problem arising is that the deformed logarithmic and exponential functions are almost, but not exactly, each others inverse. This problem is solved in the present paper.

Starting point is a discussion of properties that a generalized exponential or logarithmic function should satisfy. Functions satisfying these requirements are called $\kappa$-deformed. Next it is shown that, given a pair of 
$\kappa$-deformed logarithmic and exponential functions, one can always produce another $\kappa$-deformed logarithmic function, which will be called the deduced logarithmic function. Finally, it is shown that, if in Shannon's definition the logarithm is replaced by the deduced logarithmic function, then the equilibrium distribution is the Boltzmann-Gibbs distribution with the exponential function replaced by the $\kappa$-deformed exponential function.

\section{Properties of $\kappa$-deformed functions}

\subsection{Definitions}

The $\kappa$-deformed exponential function is denoted $\exp _{\kappa}(x)$. The deformation parameter $\kappa$ is a number not further specified. It satisfies the following assumptions

(A0) $\exp _{\kappa}(x) \geq 0$ for all real $x$. Note that $\exp _{\kappa}(x)=\infty$ is allowed.

(A1) $\exp _{\kappa}(x)$ is a convex function which is strictly increasing in all points where its value is not zero or infinite.

$(\mathrm{A} 2) \exp _{\kappa}(0)=1$.

(A3) $\exp _{\kappa}(x)$ goes fast enough to zero when $x$ goes to $-\infty$, so that

$$
\int_{0}^{\infty} \mathrm{d} x \exp _{\kappa}(-x)<\infty
$$

Sinilarly, the $\kappa$-deformed logarithm is denoted $\ln _{\kappa}(x)$. It is defined for all positive $x$ and satisfies

(B1) $\ln _{\kappa}(x)$ is a strictly increasing concave function, defined for all $x>0$.

(B2) $\ln _{\kappa}(1)=0$.

(B3) $\int_{0}^{1} \mathrm{~d} x \ln _{\kappa}(x)$ is finite.

It is easy to see that the inverse of a $\kappa$-deformed exponential function is a $\kappa$-deformed logarithm. Because $\exp _{\kappa}(x)$ is convex, and strictly increasing at $x=1$, it diverges when $x$ becomes large. Because $\exp _{\kappa}(-x)$ goes to zero when $x$ becomes large, the range of $\exp _{\kappa}(x)$ includes the interval $(0,+\infty)$. This implies that the inverse function is defined for all positive $x$. Conversely, 
if a $\kappa$-deformed logarithm is given then a $\kappa$-deformed exponential function is defined by

$$
\begin{aligned}
& \exp _{\kappa}(x)=y \quad \text { if } y>0 \text { exists for which } x=\ln _{\kappa}(y) \text { holds, } \\
& =0 \quad \text { if } x<\ln _{\kappa}(y) \text { for all } y>0 \text {, } \\
& =+\infty \text { if } x>\ln _{\kappa}(y) \text { for all } y>0 \text {. }
\end{aligned}
$$

\subsection{Dual definitions}

One property of the usual exponential function, that one may wish to hold for the deformed exponential function as well, is

$(\mathrm{A} 4) \exp _{\kappa}(-x) \exp _{\kappa}(x)=1$ whenever $\exp _{\kappa}(-x)$ and $\exp _{\kappa}(x)$ are not both zero or infinite.

The corresponding property of the logarithm is

(B4) $\ln _{\kappa}(1 / x)=-\ln _{\kappa}(x)$.

These properties are not included in the above specifications because some of the deformed functions, used as an example below, do not have them. If that is the case then one can define dual deformed functions, provided that

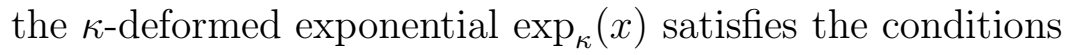

$$
\int_{0}^{+\infty} \mathrm{d} x\left(\exp _{\kappa}(x)\right)^{-1}<+\infty
$$

(A6) $\left(\exp _{\kappa}(-x)\right)^{-1}$ is a convex function of $\mathrm{x}$,

The dual deformed exponential $\exp _{\kappa}^{*}(x)$ is defined by

$$
\exp _{\kappa}^{*}(x)=\frac{1}{\exp _{\kappa}(-x)}
$$

Similarly, a dual deformed logarithm is defined by

$$
\ln _{\kappa}^{*}(x)=-\ln _{\kappa}(1 / x) .
$$

\subsection{Deduced logarithmic functions}

A notation is introduced for the integral of the $\kappa$-deformed logarithm. Let

$$
F_{\kappa}(x)=\int_{1}^{x} \mathrm{~d} y \ln _{\kappa}(y), \quad x>0
$$

This function satisfies $F_{\kappa}(x) \geq 0, F_{\kappa}(1)=0$, and $F_{\kappa}(0)<+\infty$. It is convex because the derivative $\ln _{\kappa}(x)$ is increasing. 
Introduce a new function, denoted $\omega_{\kappa}(x)$, by

$$
\omega_{\kappa}(x)=(x-1) F_{\kappa}(0)-x F_{\kappa}(1 / x) .
$$

This function is again a $\kappa$-deformed logarithm, provided that

(B5) $\int_{0}^{1} \mathrm{~d} x \ln _{\kappa}(1 / x)<+\infty$.

Note that the latter is one of the conditions for existence of the dual function $\ln _{\kappa}^{*}(x)$.

Proof:

- $\omega_{\kappa}(x)$ is a strictly increasing concave function.

To see this, first note that the integral $F_{\kappa}(x)$ of the $\kappa$-deformed logarithm $\ln _{\kappa}(x)$ can be written as

$$
F_{\kappa}(x)=x \ln _{\kappa}(x)-\int_{1}^{x} y \mathrm{~d} \ln _{\kappa}(y) .
$$

This expression is used to write the derivative of $\omega_{\kappa}(x)$ as

$$
\begin{aligned}
\frac{\mathrm{d}}{\mathrm{d} x} \omega_{\kappa}(x) & =F_{\kappa}(0)-F_{\kappa}(1 / x)+(1 / x) \ln _{\kappa}(1 / x) \\
& =\int_{0}^{1 / x} y \mathrm{~d} \ln _{\kappa}(y) .
\end{aligned}
$$

Because $\ln _{\kappa}(y)$ is a strictly increasing function the latter expression is strictly positive for all $x>0$. Hence $\omega_{\kappa}(x)$ is strictly increasing. But it is also clear from (17) that the derivative of $\omega_{\kappa}(x)$ is a decreasing function. Hence $\omega_{\kappa}(x)$ is concave.

- $\omega_{\kappa}(1)=0$.

This is obvious from the definition, using $F_{\kappa}(1)=0$.

- $\int_{0}^{1} \mathrm{~d} x \omega_{\kappa}(x)$ is finite.

One calculates, using partial integration,

$$
\begin{aligned}
\int_{0}^{1} \mathrm{~d} x \omega_{\kappa}(x)= & -F_{\kappa}(0)-\int_{0}^{1} \mathrm{~d} x x \int_{0}^{1 / x} \mathrm{~d} y \ln _{\kappa}(y) \\
= & -F_{\kappa}(0)-\frac{1}{2} \int_{0}^{1} \mathrm{~d} x\left[\frac{\mathrm{d}}{\mathrm{d} x}\left(x^{2} \int_{0}^{1 / x} \mathrm{~d} y \ln _{\kappa}(y)\right)\right. \\
& \left.+\ln _{\kappa}(1 / x)\right] \\
= & -\frac{1}{2} F_{\kappa}(0)-\frac{1}{2} \int_{0}^{1} \mathrm{~d} x \ln _{\kappa}(1 / x),
\end{aligned}
$$

which is finite by assumption (B5). 
For convenience, $\omega_{\kappa}(x)$ is called the deduced logarithmic function. The reason for introducing it is that it satisfies the equation

$$
\frac{\mathrm{d}}{\mathrm{d} x}\left(x \omega_{\kappa}(1 / x)\right)=-F_{\kappa}(0)-\ln _{\kappa}(x)
$$

equation which is used later on.

\subsection{Scaling}

For further use let us note that by scaling one can make, in a rather trivial way, new deformed functions out of existing ones.

Fix $\lambda>0$ and $\mu>0$. Given a $\kappa$-deformed logarithm $\ln _{\kappa}(x)$, a new $\kappa$-deformed logarithm $\ln _{\kappa}^{\text {scaled }}(x)$ is defined by

$$
\ln _{\kappa}^{\text {scaled }}(x)=\lambda\left(\ln _{\kappa}(\mu x)-\ln _{\kappa}(\mu)\right) .
$$

The corresponding relation between $\kappa$-deformed exponentials is

$$
\exp _{\kappa}^{\text {scaled }}(x)=\mu^{-1} \exp _{\kappa}\left(\lambda^{-1} x+\ln _{\kappa}(\mu)\right) .
$$

The deduced logarithms, if they exist, are related by

$$
\omega_{\kappa}^{\text {scaled }}(x)=\lambda\left(\omega_{\kappa}\left(\mu^{-1} x\right)-\omega_{\kappa}\left(\mu^{-1}\right)\right) .
$$

Hence the deduced logaritm is scaled with parameters $\lambda$ and $\mu^{-1}$.

\subsection{Tsallis' deformed logarithm}

Introduce the following example of $\kappa$-deformed logarithm

$$
\ln _{\kappa}(x)=\left(1+\frac{1}{\kappa}\right)\left(x^{\kappa}-1\right) .
$$

$(-1<\kappa<1)$. The inverse function is

$$
\exp _{\kappa}(x)=\left[1+\frac{\kappa}{1+\kappa} x\right]_{+}^{1 / \kappa}
$$

where $[x]_{+}=\max \{x, 0\}$. Note that for $\kappa=0$ these functions coincide with the usual definitions of logarithmic and exponential functions.

Let us verify that (13) satisfies the conditions for being a $\kappa$-deformed logarithm. 
- $\ln _{\kappa}(x)$ is a strictly increasing concave function.

One calculates

$$
\begin{aligned}
\frac{\mathrm{d}}{\mathrm{d} x} \ln _{\kappa}(x) & =(1+\kappa) x^{\kappa-1}, \\
\frac{\mathrm{d}^{2}}{\mathrm{~d} x^{2}} \ln _{\kappa}(x) & =-\left(1-\kappa^{2}\right) x^{\kappa-2} .
\end{aligned}
$$

It is obvious that the first derivative is always strictly positive, and that the second derivative is negative.

- $\ln _{\kappa}(1)=0$.

This is clear.

- $\int_{0}^{1} \mathrm{~d} x \ln _{\kappa}(x)$ is finite.

Indeed, one has $\int_{0}^{1} \mathrm{~d} x \ln _{\kappa}(x)=-1$.

This shows that the $\kappa$-deformed logarithm satisfies the assumptions.

The dual deformed logarithm exists and is given by

$$
\begin{aligned}
\ln _{\kappa}^{*}(x) & =-\ln _{\kappa}(1 / x) \\
& =\frac{1+\kappa}{1-\kappa} \ln _{-\kappa}(x) .
\end{aligned}
$$

The deduced logarithm equals the dual $\kappa$-deformed logarithm, up to scaling. To see this, first calculate

$$
\begin{aligned}
F_{\kappa}(x) & =\left(1+\frac{1}{\kappa}\right) \int_{1}^{x} \mathrm{~d} y\left(y^{\kappa}-1\right) \\
& =\frac{1}{\kappa}\left(x^{1+\kappa}-1\right)-\left(1+\frac{1}{\kappa}\right)(x-1) .
\end{aligned}
$$

In particular, one has $F_{\kappa}(0)=1$. Hence one obtains

$$
\begin{aligned}
\omega_{\kappa}(x) & =x-1-x \frac{F_{-\kappa}(1 / x)}{F_{-\kappa}(0)} \\
& =\frac{1}{\kappa}\left(1-x^{-\kappa}\right) \\
& =\frac{1}{1-\kappa} \ln _{-\kappa}(x) \\
& =\frac{1}{1+\kappa} \ln _{\kappa}^{*}(x) .
\end{aligned}
$$

One concludes that $\omega_{\kappa}(x)=\ln _{-\kappa}^{\text {scaled }}(x)$ with scaling parameters $\lambda$ and $\mu$ any pair of positive numbers satisfying $(1-\kappa) \lambda=\mu^{\kappa}$. 
The expression $\kappa^{-1}\left(1-x^{-\kappa}\right)$ has been proposed by Tsallis [1] as the definition of the deformed logarithm. In the present context it is a $\kappa$-deformed logarithm, but one which can be deduced from another one, defined by (13). Of course, the difference between both is small since $\omega_{\kappa}(x)$ is a scaled version of $\ln _{-\kappa}(x)$.

In the context of Tsallis' thermostatistics one is used to the notations $\exp _{q}(x)$ and $\ln _{q}(x)$ with $q$ related to $\kappa$ by $q=1+\kappa$.

Tsallis' definition of deformed logarithms has been studied in [6]. In Naudts and Czachor [10] a modification of (13) was proposed with the intention of making a self-dual deformed logarithm. However, the modification destroys the property of concavity, which is emphasized in the present paper.

\subsection{Kaniadakis' deformed functions}

An example of self-dual $\kappa$-deformed exponential resp. logarithmic functions is found in the work of Kaniadakis 12, 13.

Let $-1<\kappa<1, \kappa \neq 0$, and define

$$
\exp _{\kappa}(x)=\left(\kappa x+\sqrt{1+\kappa^{2} x^{2}}\right)^{1 / \kappa} .
$$

It is strictly positive and finite for all real $x$. The inverse function is

$$
\ln _{\kappa}(x)=\frac{1}{2 \kappa}\left(x^{\kappa}-x^{-\kappa}\right) .
$$

In the limit $\kappa=0$ these functions coincide with the usual definitions of logarithmic and exponential functions. It is straightforward to verify that the assumptions (A0-3) and (B1-3) are satisfied. In particular, one verifies that

$$
\int_{0}^{1} \mathrm{~d} x \ln _{\kappa}(x)=\frac{-1}{1-\kappa^{2}}
$$

and

$$
\begin{aligned}
\exp _{\kappa}(-x) \exp _{\kappa}(x) & =\left(-\kappa x+\sqrt{1+\kappa^{2} x^{2}}\right)^{1 / \kappa}\left(\kappa x+\sqrt{1+\kappa^{2} x^{2}}\right)^{1 / \kappa} \\
& =1
\end{aligned}
$$

The latter means that the functions are self-dual.

Next calculate

$$
\begin{aligned}
F_{\kappa}(x) & =\int_{1}^{x} \mathrm{~d} y \frac{1}{2 \kappa}\left(y^{\kappa}-y^{-\kappa}\right) \\
& =\frac{1}{1-\kappa^{2}}+\frac{1}{2 \kappa}\left[\frac{1}{1+\kappa} x^{1+\kappa}-\frac{1}{1-\kappa} x^{1-\kappa}\right] .
\end{aligned}
$$


In particular is

$$
F_{\kappa}(0)=\frac{1}{1-\kappa^{2}}
$$

The deduced logarithm is then given by

$$
\begin{aligned}
\omega_{\kappa}(x) & =(x-1) F_{\kappa}(0)-x F_{\kappa}(1 / x) \\
& =\frac{1}{1-\kappa^{2}}\left(-1+\frac{1+\kappa}{2 \kappa} x^{\kappa}-\frac{1-\kappa}{2 \kappa} x^{-\kappa}\right) \\
& =-\frac{1}{1-\kappa^{2}}+\frac{1}{\sqrt{1-\kappa^{2}}} \ln _{\kappa}\left(\left(\frac{1+\kappa}{1-\kappa}\right)^{1 / 2 \kappa} x\right) .
\end{aligned}
$$

Hence one has $\omega_{\kappa}(x)=\ln _{\kappa}^{\text {scaled }}(x)$ with scaling parameters

$$
\lambda=\frac{1}{\sqrt{1-\kappa^{2}}}, \quad \mu=\left(\frac{1+\kappa}{1-\kappa}\right)^{1 / 2 \kappa} .
$$

\section{Thermostatistics}

\subsection{Information content}

Following [10, 11], information content is determined by an increasing function $\omega(x)$, which is positive for $x \geq 1$. The amount of information, contained in the knowledge that event $k$ has possibility $p_{k}$, equals $\omega\left(1 / p_{k}\right)$. Hence, less probable events have a higher information content. Hartley's measure of information [1] corresponds with the choice $\omega(x)=\ln (x)$. The obvious generalization is then to take $\omega(x)$ equal to the $\kappa$-deformed logarithm. However, this turns out not to be the most convenient choice. Rather let $\omega(x)=\omega_{\kappa}(x)$ with $\omega_{\kappa}(x)$ the deduced logarithm corresponding with $\ln _{\kappa}(x)$. The average information content $I_{\kappa}(p)$ is then given by

$$
I_{\kappa}(p)=\sum_{k} p_{k} I_{k} \leq+\infty \quad \text { with } \quad I_{k}=\omega_{\kappa}\left(1 / p_{k}\right)
$$

From the definition of $\omega_{\kappa}(x)$ follows immediately that

$$
I_{\kappa}(p)=\sum_{k}\left(\left(1-p_{k}\right) F_{\kappa}(0)-F_{\kappa}\left(p_{k}\right)\right) \text {. }
$$

Because $F_{\kappa}(x)$ is convex one has

$$
\begin{aligned}
F_{\kappa}\left(p_{k}\right) & \leq p_{k} F_{\kappa}(1)+\left(1-p_{k}\right) F_{\kappa}(0) \\
& =\left(1-p_{k}\right) F_{\kappa}(0) .
\end{aligned}
$$


Hence one has always $I_{\kappa}(p) \geq 0$. This is of course also obvious from the definition (27) and the fact that $\omega_{\kappa}(x) \geq 0$ for all $x \geq 1$.

Note that $I_{\kappa}(p)=0$ if and only if $p_{k}=1$ for a single value of $k$. This follows because $F_{\kappa}(x)$ is strictly decreasing on the interval $0 \leq x \leq 1$.

$I_{\kappa}(p)$ is a concave function. This means that, if $p$ and $q$ are two probability distributions, then

$$
I_{\kappa}(\lambda p+(1-\lambda) q) \geq \lambda I_{\kappa}(p)+(1-\lambda) I_{\kappa}(q)
$$

holds for any $\lambda, 0 \leq \lambda \leq 1$. This follows immediately from (28) because $F_{\kappa}(x)$ is a convex function.

\subsection{Examples}

If $\omega_{\kappa}(x)$ coincides with the usual logarithmic function then one finds

$$
I_{\kappa}(p)=-\sum_{k} p_{k} \ln p_{k}
$$

This is Shannon's expression for information content [2].

Now if $\omega_{\kappa}(x)$ equals Tsallis' deformed logarithm, as given in (18), then average information content equals

$$
I_{\kappa}^{\text {Tsallis }}(p)=\frac{1}{\kappa}\left(1-\sum_{k} p_{k}^{1+\kappa}\right) .
$$

This is the entropy functional used in Tsallis' thermostatistics. On the other hand, if $\omega_{\kappa}(x)$ is given by (25), the logarithmic function deduced from Kaniadakis' deformed logarithm, then one obtains

$$
\begin{aligned}
I_{\kappa}(p) & =\frac{1}{2 \kappa(1-\kappa)}\left(\sum_{k} p_{k}^{1-\kappa}-1\right)+\frac{1}{2 \kappa(1+\kappa)}\left(1-\sum_{k} p_{k}^{1+\kappa}\right) \\
& =\frac{1}{2(1+\kappa)} I_{\kappa}^{\text {Tsallis }}(p)+\frac{1}{2(1-\kappa)} I_{-\kappa}^{\text {Tsallis }}(p) .
\end{aligned}
$$

This expression differs slightly from the one proposed in [12]. The reason is that here information content is defined using the deduced logarithm $\omega_{\kappa}(x)$ instead of the deformed logarithm $\ln _{\kappa}(x)$. As discussed before, $\omega_{\kappa}(x)$ is a scaled version of $\ln _{\kappa}(x)$.

\subsection{Variational principle}

Let us optimize information content $I_{\kappa}(p)$ under a linear constraint

$$
\sum_{k} p_{k} E_{k}=U
$$


where $E_{k}$ is a sequence of energies, bounded from below, and $U$ is a target value of the average energy. It is tradition to solve this problem by introducing Lagrange multipliers $\alpha$ and $\beta$ to control the normalization $\sum_{k} p_{k}=1$ and the constraint (34). Variation of

$$
I_{\kappa}(p)-\alpha \sum_{k} p_{k}-\beta \sum_{k} p_{k} E_{k}
$$

with respect to $p_{k}$ gives, using (9),

$$
0=-F_{\kappa}(0)-\ln _{\kappa}\left(p_{k}\right)-\alpha-\beta E_{k}
$$

This expression can be written as

$$
p_{k}=\exp _{\kappa}\left(-F_{\kappa}(0)-\alpha-\beta E_{k}\right),
$$

and is clearly a generalization of the well-known Boltzmann-Gibbs equilibrium distribution. The parameter $\alpha$ should be chosen in such a way that

$$
1=\sum_{k} \exp _{\kappa}\left(-F_{\kappa}(0)-\alpha-\beta E_{k}\right)
$$

holds. Because $\exp _{\kappa}$ is a strictly increasing function this equation has atmost one solution. Whether it has a solution at all depends on wether the sum in the r.h.s. of (38) converges. This will be the case when the sum contains a finite number of terms or when the energies $E_{k}$ increase fast enough with $k$.

\subsection{Examples}

Let $\gamma=-\kappa\left(F_{\kappa}(0)+\alpha\right)$. In the case of Tsallis' deformed functions (37) becomes

$$
p_{k}=(1+\kappa)^{-1 / \kappa}\left[1+\kappa+\gamma-\kappa \beta E_{k}\right]_{+}^{1 / \kappa} .
$$

This is the typical form of the equilibrium probability distribution in Tsallis' thermostatistics. In particular, the probabilities $p_{k}$ vanish exactly when the expression inside the brackets $[\cdots]_{+}$becomes negative. On the other hand, using Kaniadakis' definitions one obtains

$$
p_{k}=\left(\gamma-\kappa \beta E_{k}+\sqrt{1+\left(\gamma-\kappa \beta E_{k}\right)^{2}}\right)^{1 / \kappa} .
$$

In this case the probabilities $p_{k}$ are always strictly positive. 


\subsection{Discussion}

Generic existence and uniqueness of solutions of the variational problem (35) still has to be studied. In the case of Tsallis' thermostatistics, these mathematical aspects have been studied in [5, 8, 9]. Because of the concavity of average information content $I_{\kappa}(p)$, and relation (9) between the $\kappa$-deformed logarithm and the deduced logarithm, one can expect that it will be feasible, not only to generalize these results, but also to simplify the proofs.

In Tsallis' thermostatistics the constraint (34) is not the only one that is considered. The constraint $\sum_{k} p_{k}^{q} E_{k}=U$ has been used some time, but its justification on physical grounds seems to be missing. In [7] the constraint

$$
\frac{\sum_{k} p_{k}^{q} E_{k}}{\sum_{k} p_{k}^{q}}=U
$$

has been introduced. By introduction of the so-called escorte probabilities

$$
P_{k}=\frac{p_{k}^{q}}{\sum_{k} p_{k}^{q}}
$$

it transforms into a constraint of the usual form (34). Let $\kappa^{\prime}=-\kappa /(1+\kappa)$. With $q=1+\kappa$ and $q^{\prime}=1+\kappa^{\prime}$ this relation reads $q^{\prime}=1 / q$. Because of the identity

$$
\left(1-\kappa^{\prime} I_{\kappa^{\prime}}^{\text {Tsallis }}(P)\right)^{1 / \kappa^{\prime}}\left(1-\kappa I_{\kappa}^{\text {Tsallis }}(p)\right)^{1 / \kappa}=1,
$$

maximizing information content $I_{\kappa}^{\text {Tsallis }}(p)$ with constraint (41) is equivalent with maximizing $I_{\kappa^{\prime}}^{\text {Tsallis }}(P)$ using the constraint $\sum_{k} P_{k} E_{k}=U$. Hence both formalisms are equivalent, as is well-known. However, from $\kappa<1$ follows the condition that $\kappa^{\prime}>-1 / 2$. Conversely, from $\kappa^{\prime}<1$ follows the condition that $\kappa>-1 / 2$. These conditions are needed here to guarantee that the $\kappa$ deformed exponential and logarithmic functions have their basic properties. One concludes that for $\kappa$ in the range $-1<\kappa<-1 / 2$ the optimization problem with constraint (41) is not covered by the analysis of the problem with constraint (34).

In 110, 11 constraints are considered which involve nonlinear averages of the Kolmogorov-Nagumo type. In particular, a class of optimization problems was studied that could be transformed into optimization problems of the Tsallis type. In this way a generalized thermostatistics was formulated. By using the results of the present paper this thermostatistics can be generalized even further. By doing so, the limits of generalizing the Boltzmann-Gibbs formalism come into sight. Such an effort is however out of scope of the present paper. 


\section{References}

[1] R.V. Hartley, Transmission of information, Bell System Technical Journal, 7, 1928.

[2] C.E. Shannon, Bell System Technical J. 27, 379 (1948); 27, 623 (1948).

[3] C. Tsallis, Possible Generalization of Boltzmann-Gibbs Statistics, J. Stat. Phys. 52, 479-487 (1988).

[4] C. Tsallis, What are the numbers that experiments provide? Quimica Nova 17, 468 (1994)

[5] G.R. Guerberoff and G.A. Raggio, Standard thermal statistics with q-entropies, J. Math. Phys. 37(4), 1776-1789 (1996).

[6] E.P. Borges, On a q-generalization of circular and hyperbolic functions, J. Phys. A 31, 5281-5288 (1998).

[7] C. Tsallis, R.S. Mendes, A.R. Plastino, The role of constraints within generalized nonextensive statistics, Physica A261, 543-554 (1998).

[8] J. Naudts and M. Czachor, Dynamic and thermodynamic stability of non-extensive systems, in: "Nonextensive Statistical Mechanics and Its Applications", ed. S. Abe, Y. Okamoto, Lecture Notes in Physics 560 (Springer-Verlag, 2001), p. 243-252.

[9] J. Naudts, Rigorous results in non-extensive thermodynamics, math-ph/9908025, Rev. Math. Phys., 12(10), 1305-1324 (2000).

[10] J. Naudts and M. Czachor, Generalized thermostatistics and Kolmogorov-Nagumo averages, cond-mat/0110077.

[11] M. Czachor and J. Naudts, Thermostatistics based on Kolmogorov-Nagumo averages: Unifying framework for extensive and nonextensive generalizations, cond-mat/0106324, to appear in Phys. Lett. A.

[12] G. Kaniadakis, Non-linear kinetics underlying generalized statistics, Physica A296, 405-425 (2001).

[13] G. Kaniadakis and A.M. Scarfone, A new one parameter deformation of the exponential function, NEXT2001 Meeting, cond-mat/0109537. 